\title{
Adeno-associated viral vectors at the frontier between tolerance and immunity
}

\author{
Federico Mingozzi ${ }^{1,2}$ * and Hildegard Büning ${ }^{3,4,5}$ * \\ ' Généthon, Evry, France \\ 2 INSERM U974, Université Pierre et Marie Curie (Paris 6), Paris, France \\ ${ }^{3}$ Center for Molecular Medicine Cologne (CMMC), University of Cologne, Cologne, Germany \\ ${ }^{4}$ German Center for Infection Research (DZIF), Partner Site Bonn-Cologne, Bonn, Germany \\ ${ }^{5}$ Department I of Internal Medicine, University Hospital of Cologne, Cologne, Germany \\ *Correspondence: fmingozzi@genethon.fr; hildegard.buening@uk-koeln.de \\ Edited and reviewed by: \\ lan Marriott, University of North Carolina at Charlotte, USA
}

Keywords: AAV vector, immune response, tolerance, vaccine, gene therapy

In recent years, the field of in vivo gene transfer with adenoassociated virus (AAV) vectors has seen an extraordinary expansion of applications and investments. Results emerging from clinical trials (1) and the recent market approval of a gene therapy drug for lipoprotein lipase deficiency (2) contributed to the hype around this vector system (3). Indeed, AAV vectors have several features that make them an ideal tool for gene transfer, for example, parental virions are replication deficient and non-pathogenic (4), and vectors can drive expression of a transgene for several years $(5,6)$ despite the fact that they do not integrate efficiently into the host genome. In recent years, a portfolio of natural AAV isolates (AAV serotypes) differing in tissue tropism has been developed as vectors. This toolbox has been further expanded with engineered AAV capsids developed to enhance efficiency and specificity of gene delivery, and to escape antibody neutralization (7). At the vector genome level, availability of potent promoter/enhancer sequences, codon-optimization of transgenes, and development of self-complementary AAV vectors (8) further enhanced efficacy of gene transfer. Finally, the availability of scalable processes to produce AAV vectors in GMP contributed significantly to the expansion of the field.

As the AAV vector technology reached a more mature stage, it has become clear that a better understanding of the interactions of viral vectors with the host immune system is needed. In this Research Topic of Frontiers in Immunology, the editors present a collection of reviews and research articles discussing the two sides of immune responses triggered by in vivo gene transfer. These responses in fact can be desirable when they result in induction of tolerance to the therapeutic transgene (9), or when they are exploited for vaccine development, as discussed by Nieto and Salvetti in their review article (10). Conversely, immunogenicity of the viral capsid or the transgene product can be detrimental, as it may result in lack or loss of efficacy following vector-mediated gene transfer.

Evidence for the critical role of tolerance induction in the achievement of sustained therapeutic efficacy following gene transfer comes from the work of Liao and colleagues, which provides evidence that glucocorticoid-induced TNF receptor (GITR) and its ligand GITR-L are of fundamental importance for the induction of immune regulatory responses in gene transfer and that lack of expression of GITR-L on antigen presenting cells results in impaired induction of regulatory T cells (Tregs) (11). Indeed, evidence of the key function of Tregs for successful in vivo gene therapy comes from several studies (12), and Liu and colleagues further demonstrate this concept in a model of plasmid gene transfer for hemophilia $\mathrm{A}$, in which a combination of $\mathrm{B}$ cell depleting and Treg-enhancing drugs is used to successfully modulate transgene immunogenicity (13).

\section{INNATE IMMUNE RESPONSES TO AAV VECTORS}

The innate immune system constitutes the first line of defense against invading pathogens. It recognizes evolutionarily conserved structures foreign to the host or detects structures known as self, but present in the wrong intracellular compartment, via innate immune sensors termed pathogen recognition receptors (PRRs). Binding of such pathogen-associated molecular patterns (PAMPs) to PRRs activates the intracellular innate immune system, leading to substantial changes in the expression of genes related to host defense, in secretion of cytokines and chemokines, and up-regulation of co-stimulatory molecules, which as a consequence induce or modulate the adaptive arm of the immune system.

Of the four families of cellular PRRs [toll-like-receptors (TLRs), NOD-like receptors, RIG-like receptors, and C-type lectin receptors], as of now only two members of the TLR family, TLR-2 and TLR-9, have been described as sensors for AAV vectors. TLR-2 was identified as a PRR of the viral capsid in studies on cell autonomous immune responses in primary human liver cells (liver sinusoidal endothelial cells, Kupffer cells) and activated endothelial cells (14), while TLR-9 was reported as sensor of AAV vector genomes in plasmacytoid dendritic cells ( $\mathrm{pDC}$ ) isolated from mice and humans (15). Although both PRRs are part of the same family, recognition of the viral capsid caused induction of a Nuclear Factor kB-dependent inflammatory response (14), while activation of TLR-9 induced secretion of type I interferon (IFN) that was found to be enhanced if vectors with self-complementary (sc) AAV vector genomes were used $(15,16)$. The nature of this enhanced immunogenicity remains to be clarified, but is maybe related to a negative impact of sc vector genomes on capsid stability (16) or to the additional inverted terminal repeat (ITR) sequence present in sc vector genomes (8). The later hypothesis would be in line with a recent study reporting significantly reduced adaptive 
immune responses toward the capsid and the transgene product when using AAV vectors with a reduced number of $\mathrm{CpG}$ motifs, which are known TLR-9 PAMPs (17). The route of vector delivery appears to be a critical factor in AAV recognition by the innate immune system. The above-described activation of the TLR-9 myeloid differentiation primary response 88 (MyD88) signaling pathway, for example, resulted in humoral and $\mathrm{T}$ cell-mediated adaptive immune responses toward the AAV capsid and the transgene product in mice in which AAV vectors were administered intramuscularly. Conversely, following tail vein injection, neither a TLR-2, nor a TLR-9, or a type I IFN dependent induction of AAV specific IgG antibodies could be detected $(15,18)$.

\section{ADAPTIVE IMMUNE RESPONSES IN AAV VECTOR-MEDIATED GENE TRANSFER}

Exposure to wild-type AAV or to AAV vectors, and the consequent activation of innate and adaptive immunity to vector and transgene leads to both antibody and cell-mediated responses. Antibodies directed against the AAV capsid are highly prevalent in humans (up to $60 \%$ of healthy individuals) and can efficiently neutralize the vector even when present at low titers, resulting in lack of efficacy, thus posing a significant constrain to patients enrollment in clinical trials. Similarly, vector administration results in long-lasting high-titer anti-AAV neutralizing antibodies (NAb), which prevent vector readministration. Results from human trials and studies conducted in small and large animal models of gene transfer showed that NAb titers as low as 1:5 can completely block $\mathrm{AAV}$ vector transduction, and that $\mathrm{AAV}$ vectors remain susceptible to antibody-mediated neutralization for several hours after intravascular delivery.

Two contributions on the topic of anti-AAV antibodies can be found in this Research Topic. Calcedo and Wilson reviewed the issue of $\mathrm{NAb}$ directed against $\mathrm{AAV}$; in their manuscript, they discussed the prevalence of NAb in various human populations, the issue of antibody cross-reactivity, and finally the assays used to measure antibodies to AAV, and the strategies that could possibly be used to overcome this limitation (19). In the second review article, Tseng and Agbandje-McKenna (20) discuss different approaches to antibody epitope mapping and the relationship of these epitopes with the capsid structure. Furthermore, they suggest how this knowledge can be exploited to drive the efforts toward engineering novel AAV capsid variants resistant to antibodies, and to gain a better understanding on the structure-function-relationship across AAV serotypes when it comes to the interactions with the immune system.

In addition to neutralizing antibodies, natural infection with wild-type AAV also triggers cell-mediated immune responses against the capsid, which results in a reservoir of memory $\mathrm{CD}^{+}$ $\mathrm{T}$ cells that can be reactivated upon vector administration. This can cause the destruction of transduced cells harboring AAV capsid antigen in the context of MHC class I, as it has been observed in subjects enrolled in AAV vector-mediated liver gene transfer trials. Several questions remain on the role of these capsid-specific $\mathrm{CD}^{+} \mathrm{T}$ cells in the outcome of gene transfer, as detection of $\mathrm{T}$ cell reactivity to the capsid in PBMCs has not always been associated with detrimental effects on gene transfer in liver and muscle trials. Notably, experience from the AAV8 gene therapy trials in hemophilia B subjects suggests that timely administration of immunosuppression can prevent detrimental effects of capsid-directed $\mathrm{T}$ cell immunity.

Three review articles in this Research Topic focus specifically on adaptive immune responses to AAV vectors in the context of gene transfer to different tissues, and discuss the issue of $\mathrm{T}$ cellmediated immunity directed against the vector capsid. Willett and Bennett provide an overview of what it is known about gene transfer in an immune privileged body site, the eye, describing the unique and valuable lessons learned from the preclinical and clinical studies of AAV gene transfer for RPE65 deficiency (21). Ferreira and colleagues describe the experience with AAV vectors in muscle gene transfer in the context of the development of Glybera, the approved drug for the treatment of lipoprotein lipase deficiency (22). Finally, in their manuscript, Basner-Tschakarjan and Mingozzi provide a broad overview on the issue of $\mathrm{T}$ cell immunity to AAV vectors focusing on data emerging from gene therapy trials (23). To complete this collection of articles on immune responses in gene transfer, two review articles discuss the tools available to the investigators to study the immunogenicity of AAV vectors. Basner-Tschakarjan and colleagues provide an overview of in vitro and in vivo preclinical models that have helped to explain the immune responses to AAV vectors observed in human trials (24), while Britten and colleagues address the extremely important issue of immune assay standardization in clinical trials (25).

\section{ACKNOWLEDGMENTS}

This work was supported by the German Research Foundation (SFB670, TP8; to HB), the Center for Molecular Medicine Cologne (C1; to HB), by Genethon (to FM), by the European Union (FP7PEOPLE-2012-CIG Grant 333628; ERC-2013-CoG grant 617432; to FM), and the Bayer Early Career Investigator Award (to FM).

\section{REFERENCES}

1. Mingozzi F, High KA. Therapeutic in vivo gene transfer for genetic disease using AAV: progress and challenges. Nat Rev Genet (2011) 12:341-55. doi: $10.1038 / \operatorname{nrg} 2988$

2. Buning H. Gene therapy enters the pharma market: the short story of a long journey. EMBO Mol Med (2013) 5:1-3. doi:10.1002/emmm.201202291

3. Gormley B. Therapies Push Investors to Examine New Areas of Human Body. Wall Street Journal Venture Capital Dispatch (2014). Available from: http:// blogs.wsj.com/venturecapital/2014/09/14/gene-therapies-push-investors-toexamine-new-areas-of-human-body/

4. Muzyczka N, Berns KI. Parvoviridae: The Viruses and their Replication. Edn. 4th ed. Philadelphia, PA: Lippincott, Williams and Wilkins (2001).

5. Buchlis G, Podsakoff GM, Radu A, Hawk SM, Flake AW, Mingozzi F, et al. Factor IX expression in skeletal muscle of a severe hemophilia B patient 10 years after AAV-mediated gene transfer. Blood (2012) 119:3038-41. doi:10.1182/blood2011-09-382317

6. Nathwani AC, Reiss UM, Tuddenham EG, Rosales C, Chowdary P, McIntosh J, et al. Long-term safety and efficacy of factor IX gene therapy in hemophilia B. N Engl J Med (2014) 371:1994-2004. doi:10.1056/NEJMoa1407309

7. Bartel M, Schaffer D, Buning H. Enhancing the clinical potential of AAV vectors by capsid engineering to evade pre-existing immunity. Front Microbiol (2011) 2:204. doi:10.3389/fmicb.2011.00204

8. McCarty DM, Fu H, Monahan PE, Toulson CE, Naik P, Samulski RJ. Adenoassociated virus terminal repeat (TR) mutant generates self-complementary vectors to overcome the rate-limiting step to transduction in vivo. Gene Ther (2003) 10:2112-8. doi:10.1038/sj.gt.3302134 
9. Mingozzi F, Liu YL, Dobrzynski E, Kaufhold A, Liu JH, Wang Y, et al. Induction of immune tolerance to coagulation factor IX antigen by in vivo hepatic gene transfer. J Clin Invest (2003) 111:1347-56. doi:10.1172/JCI200316887

10. Nieto K, Salvetti AAAV. Vectors vaccines against infectious diseases. Front Immunol (2014) 5:5. doi:10.3389/fimmu.2014.00005

11. Liao G, O’Keeffe MS, Wang G, van Driel B, de Waal Malefyt R, Reinecker HC, et al. Glucocorticoid-induced TNF receptor family-related protein ligand is requisite for optimal functioning of regulatory CD4(+) T cells. Front Immunol (2014) 5:35. doi:10.3389/fimmu.2014.00035

12. Cao O, Furlan-Freguia C, Arruda VR, Herzog RW. Emerging role of regulatory T cells in gene transfer. Curr Gene Ther (2007) 7:381-90. doi:10.2174/ 156652307782151506

13. Liu CL, Ye P, Lin J, Butts CL, Miao CH. Anti-CD20 as the B-Cell targeting agent in a combined therapy to modulate anti-factor VIII immune responses in hemophilia a inhibitor mice. Front Immunol (2014) 4:502. doi:10.3389/fimmu.2013. 00502

14. Hösel M, Broxtermann M, Janicki H, Esser K, Arzberger S, Hartmann P, et al. Toll-like receptor 2-mediated innate immune response in human nonparenchymal liver cells toward adeno-associated viral vectors. Hepatology (2012) 55:287-97. doi:10.1002/hep.24625

15. Zhu J, Huang X, Yang Y. The TLR9-MyD88 pathway is critical for adaptive immune responses to adeno-associated virus gene therapy vectors in mice. J Clin Invest (2009) 119:2388-98. doi:10.1172/JCI37607

16. Martino AT, Suzuki M, Markusic DM, Zolotukhin I, Ryals RC, Moghimi B, et al. The genome of self-complementary adeno-associated viral vectors increases Toll-like receptor 9-dependent innate immune responses in the liver. Blood (2011) 117:6459-68. doi:10.1182/blood-2010-10-314518

17. Faust SM, Bell P, Cutler BJ, Ashley SN, Zhu Y, Rabinowitz JE, et al. CpG-depleted adeno-associated virus vectors evade immune detection. J Clin Invest (2013) 123:2994-3001. doi:10.1172/JCI68205

18. Sudres M, Ciré S, Vasseur V, Brault L, Da Rocha S, Boisgérault F, et al. MyD88 signaling in B cells regulates the production of Th1-dependent antibodies to AAV. Mol Ther (2012) 20:1571-81. doi:10.1038/mt.2012.101

19. Calcedo R, Wilson JM. Humoral immune response to AAV. Front Immunol (2013) 4:341. doi:10.3389/fimmu.2013.00341
20. Tseng YS, Agbandje-McKenna M. Mapping the AAV capsid host antibody response toward the development of second generation gene delivery vectors. Front Immunol (2014) 5:9. doi:10.3389/fimmu.2014.00009

21. Willett K, Bennett J. Immunology of AAV-mediated gene transfer in the eye. Front Immunol (2013) 4:261. doi:10.3389/fimmu.2013.00261

22. Ferreira V, Petry H, Salmon F. Immune responses to AAV-vectors, the glybera example from bench to bedside. Front Immunol (2014) 5:82. doi:10.3389/fimmu. 2014.00082

23. Basner-Tschakarjan E, Mingozzi F. Cell-mediated immunity to AAV vectors, evolving concepts and potential solutions. Front Immunol (2014) 5:350. doi:10.3389/fimmu.2014.00350

24. Basner-Tschakarjan E, Bijjiga E, Martino AT. Pre-clinical assessment of immune responses to adeno-associated virus (AAV) vectors. Front Immunol (2014) 5:28. doi:10.3389/fimmu.2014.00028

25. Britten CM, Walter S, Janetzki S. Immunological monitoring to rationally guide AAV gene therapy. Front Immunol (2013) 4:273. doi:10.3389/fimmu.2013.00273

Conflict of Interest Statement: The authors declare that the research was conducted in the absence of any commercial or financial relationships that could be construed as a potential conflict of interest.

Received: 16 February 2015; accepted: 03 March 2015; published online: 17 March 2015.

Citation: Mingozzi $F$ and Büning $H$ (2015) Adeno-associated viral vectors at the frontier between tolerance and immunity. Front. Immunol. 6:120. doi: 10.3389/fimmu.2015.00120

This article was submitted to Microbial Immunology, a section of the journal Frontiers in Immunology.

Copyright (C) 2015 Mingozzi and Büning. This is an open-access article distributed under the terms of the Creative Commons Attribution License (CC BY). The use, distribution or reproduction in other forums is permitted, provided the original author(s) or licensor are credited and that the original publication in this journal is cited, in accordance with accepted academic practice. No use, distribution or reproduction is permitted which does not comply with these terms. 